Supporting information for:

\title{
Confined Water Vapor in ZIF-8 Nanopores
}

Li Zhang, Bin Zheng*, Ying Gao, Lianli Wang, Jinlei Wang, Xiaobo Duan*

School of Materials Science and Engineering, Xi'an University of Science and Technology, Xi'an 710054, PR China

Email: zhengbin@xust.edu.cn; xiaobo12558@163.com 

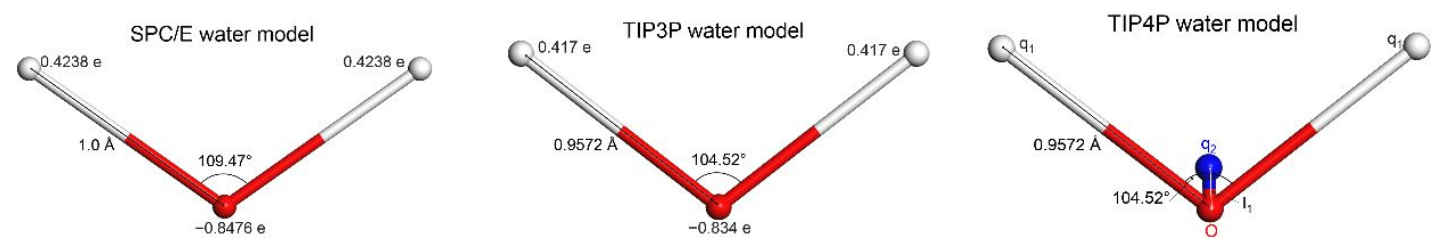

Figure S1. Water model used in this study.

Table S1. TIP4P water models used in this study.

\begin{tabular}{cccccc}
\hline & \multicolumn{2}{c}{ O atom } & \multirow{2}{*}{$\mathrm{q}_{1}(\mathrm{e})$} & $\mathrm{q}_{2}(\mathrm{e})$ & $\mathrm{1}_{1}(\AA)$ \\
\cline { 2 - 4 } & $\varepsilon^{\mathrm{a}}(\mathrm{kJ} / \mathrm{mol})$ & $\sigma^{\mathrm{b}}(\AA)$ & & -1.0484 & 0.1250 \\
\cline { 2 - 5 } TIP4P-Ew & 0.6809 & 3.164 & 0.5242 & -1.1128 & 0.1546 \\
TIP4P/2005 & 0.7749 & 3.159 & 0.5564 & -1.1794 & 0.1577 \\
TIP4P/Ice & 0.8822 & 3.167 & 0.5897 & -104 \\
\hline
\end{tabular}

aThe well depth (12-6 Lennard-Jones model) at which the strongest van der Waals attraction between two particles.

${ }^{\text {b}}$ The length (12-6 Lennard-Jones model) at which the van der Waals interaction between the two particles is zero.

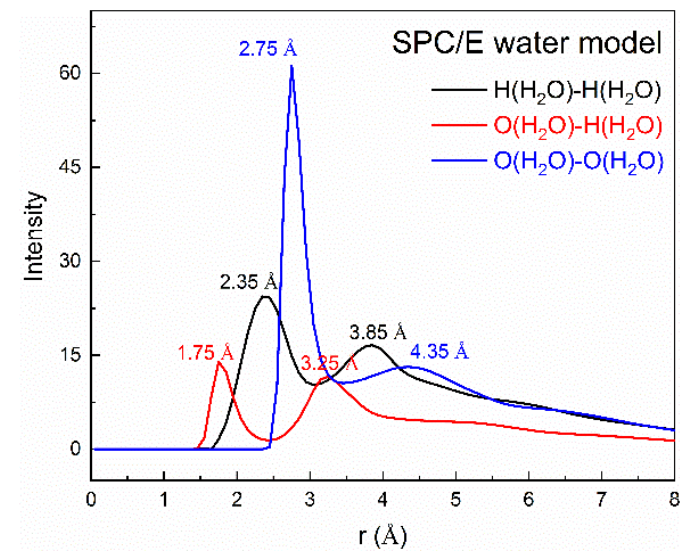

(a)

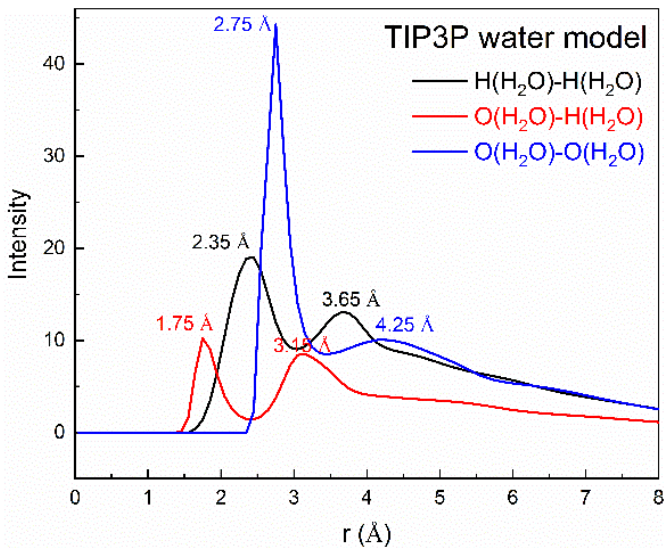

(b)

Figure S2. Radial distribution functions for water structure inside ZIF-8 nanopores. (a) SPCE/E water model and (b) TIP3P water model. A binning of $0.2 \AA$ was used here. 


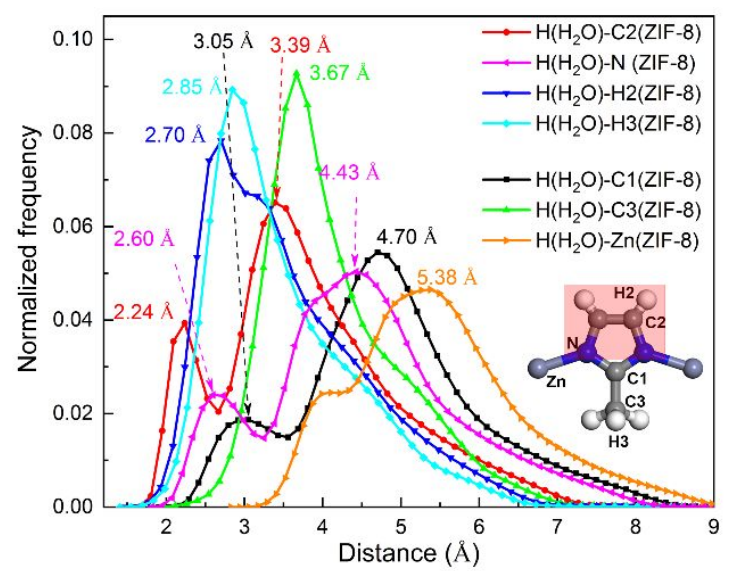

(a)

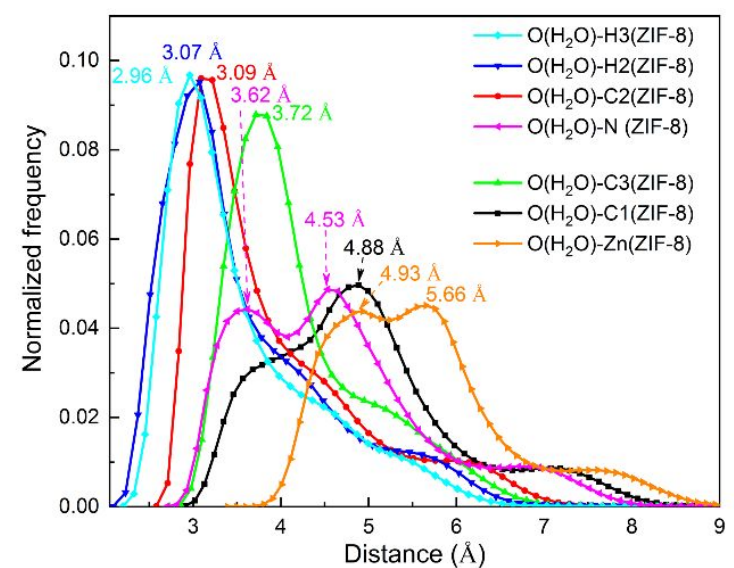

(b)

Figure S3. Distribution of the nearest distance (computed by Equation 1) between the water guest and ZIF-8 host atoms. (a) and (b) correspond to $\mathrm{H}$ and $\mathrm{O}$ atom of the water molecule, respectively. ZIF-8 atomic labels are shown in the inset picture.

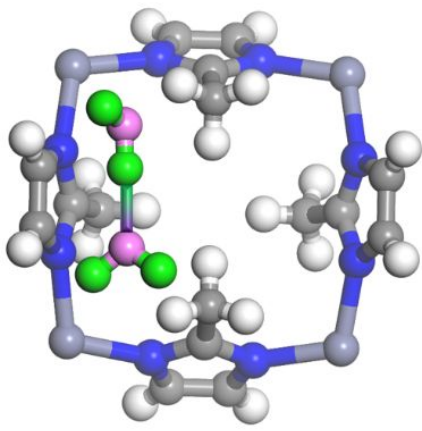

Top view

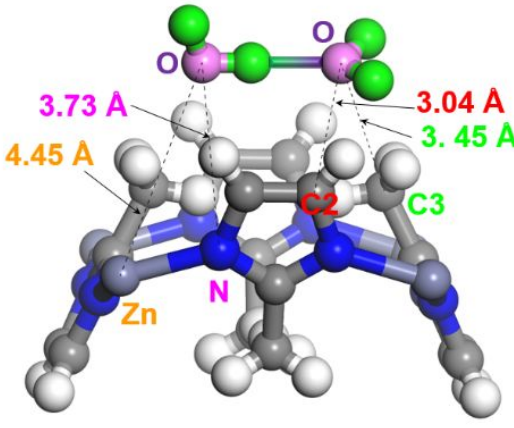

Side view

Figure S4. Possible adsorption site I for water along the 4-ring-member gate of ZIF-8 nanopores.

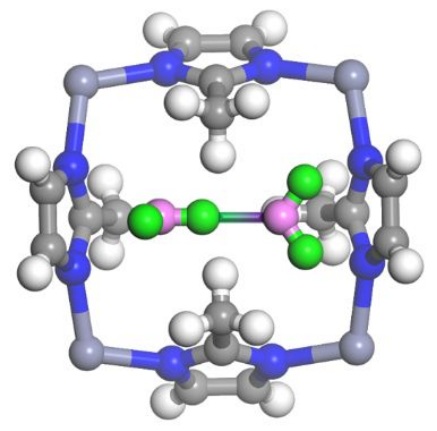

Top view

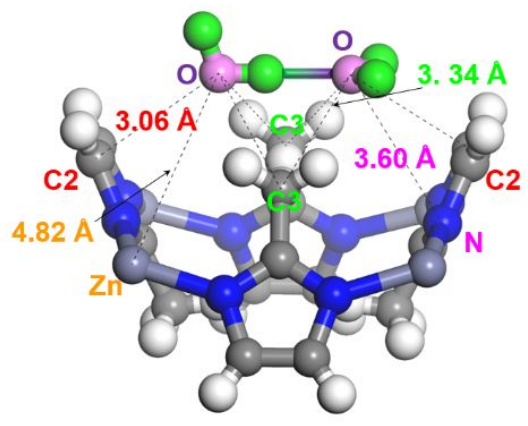

Side view

Figure S5. Possible adsorption site II for water along the 4-ring-member gate of ZIF-8 nanopores. 